\title{
Melatonin Ameliorates Bile Duct Ligation-Induced Systemic Oxidative Stress and Spatial Memory Deficits in Developing Rats
}

\author{
LI-TUNG HUANG, MAO-MENG TIAO, YOU-LIN TAIN, CHIH-CHENG CHEN, AND CHIH-SUNG HSIEH
}

Department of Pediatrics [L.-T.H., M.-M.T., Y.-L.T., C.-C.C.], Chang Gung Memorial Hospital-Kaohsiung Medical Center, Chang Gung University College of Medicine, Kaohsiung 833, Taiwan; Department of Pediatric Surgery [C.-S.H.], Pingtung Christian Hospital,

Pingtung, 900, Taiwan

\begin{abstract}
Bile duct ligation (BDL) induces primary biliary cirrhosis characterized by cholestasis, impaired liver function, and cognition. Young male Sprague-Dawley rats were used: rats underwent laparotomy without BDL [sham-control (SC) group]; rats had restricted diets supply [diet-control (DC) group]; rats underwent BDL for $2 \mathrm{wk}$ (BDL group); BDL rats with melatonin $(500 \mu \mathrm{g} / \mathrm{kg} / \mathrm{d})$ intraperitoneally for $2 \mathrm{wk}$ [melatonin $(500 \mu \mathrm{g} / \mathrm{kg} / \mathrm{d})$ (M500) group]; and BDL rats with melatonin (1000 $\mu \mathrm{g} / \mathrm{kg} / \mathrm{d} /$ intraperitoneally) for 2 wk [melatonin $(1000 \mu \mathrm{g} / \mathrm{kg} / \mathrm{d})$ (M1000) group]. All the surviving rats were assessed for spatial memory and blood was tested for biochemical study. Liver, brain cortex, and hippocampus were collected for determination of malondialdehyde (MDA) and glutathione (GSH)/ oxidized glutathione (GSSG) ratios. BDL group rats had significantly higher plasma direct/total bilirubin, aspartate aminotransferase (AST), alanine aminotransferase (ALT), MDA values and higher liver MDA values and lower GSH/GSSG ratios when compared with $\mathrm{SC}$ group. In addition, BDL group rats had impaired spatial performance. After melatonin treatment, cholestatic rats' plasma MDA levels, liver MDA levels, and liver GSH/GSSG ratios approached to the values of SC group. Only high dose of melatonin improved spatial performance. Results of this study indicate cholestasis in the developing rats increase oxidative stress and cause spatial memory deficits, which are prevented by melatonin treatment. (Pediatr Res 65: 176-180, 2009)
\end{abstract}

$\mathrm{C}$ holestasis is encountered in a variety of clinical disorders. It is the main feature of a number of chronic progressive liver diseases, including primary biliary cirrhosis, primary sclerosing cholangitis, allograft rejection, iatrogenic obstruction of bile ducts, and biliary atresia. Cholestasis is now recognized as a disorder characterized by liver oxidants overload (1-3). Furthermore, the oxidative stress in cholestatic liver disease is a systemic phenomenon $(4,5)$, probably encompassing all tissues and organs, even those separated by the blood-brain barrier (6). Similarly, oxidative stress plays an important role in the pathogenesis of toxic liver injury and other hepatic disorders (7).

Cognitive function is impaired in patients with liver disease $(8,9)$ and in animal models of chronic liver failure $(10)$. Liver failure can alter glutamatergic neurotransmission and in some

Received June 25, 2008; accepted August 19, 2008.

Correspondence: Chih-Sung Hsieh, M.D., 60 Da Lien Road, Pingtung, 900, Taiwan; e-mail: yenmeichang@yahoo.com.tw

Supported in part by grants from Chang Gung Memorial Hospital, Kaohsiung, Taiwan CMRPG850211 (L.-T.H.), CMRPG850221 (Y.-L.T.), CMRPG870561 (C.-C.C.), and CMRPG870561 (C.-S.H.). associated signal transduction pathways, including the glutamate-nitric oxide-cGMP (11) and long-term potentiation (LTP) (12). Previous studies have shown that the ability to learn the Morris water maze task is impaired in adult rats after bile duct ligation (BDL) (13), portacaval shunt $(14,15)$, portal hypertension, and thioacetamide intoxication (15). Because cognitive abilities are developmentally regulated (16), the hypothesis that BDL rats will develop spatial memory deficits has never been examined and its underlying molecular mechanisms need clarification.

Melatonin was recently discovered to be an effective free radical scavenger and antioxidant (17-19). Melatonin can decrease oxidative injury and was found to be protective after experimental traumatic brain injury (20), hypoxic brain damage (21), and seizure-induced lipid peroxidation (22). Moreover, melatonin can prevent cholestasis-induced renal (23), liver (24-26), and brain damage (27). In addition, melatonin can pass blood-brain barrier and has been safe in children and is widely used in clinical conditions (28).

The study was therefore designed to address two specific questions: First, to examine the systemic oxidative stress status and spatial performance in developing rat with BDLinduced cholestasis; Second, to test whether melatonin protects damage from systemic oxidative stress and improve spatial memory deficits caused by BDL-induced cholestasis.

\section{METHODS}

Animals. This experiment was performed under the Guidelines for Animal Experiments of Chang Gung Memorial Hospital and Chang Gung University. Totally, 12 litters were used. Within each litter, rats were randomly assigned to each group. To avoid the sex effects on spatial memory and oxidative stress, only male rats were used. Attempts were made to minimize the numbers of animals used. All animals were housed in a room maintained at $24^{\circ} \mathrm{C}$ with 12-h light/dark cycles. All animals, except those under diet restriction, had free access to standard laboratory chow.

Experimental design. Male Sprague-Dawley rats postnatal day (PND) $17 \pm 1$, weighing approximately $50 \mathrm{~g}$, underwent ketamine $(50 \mathrm{mg} / \mathrm{kg})$ and xylazine $(23 \mathrm{mg} / \mathrm{kg})$ anesthesia and received BDL as previously described (13). Briefly, obstructive jaundice was induced by opening a midline incision, and the common bile duct was ligated and divided with double ligatures of proximal duct. These experimental rats will be designated as BDL group $(n=$

Abbreviations: ALT, alanine aminotransferase; AST, aspartate aminotransferase; BDL, bile duct ligation; DC, diet-control; GSH, glutathione; GSSG, oxidized glutathione; LTP, long-term potentiation; M500, melatonin (500 $\mu \mathrm{g} / \mathrm{kg} / \mathrm{d})$; M1000, melatonin (1000 $\mu \mathrm{g} / \mathrm{kg} / \mathrm{d})$; MDA, malondialdehyde; PND, postnatal day; ROS, reactive oxygen species; SC, sham-control 
13). In addition, rats received sham ligation of bile duct as the sham-control group (SC) $(n=13)$. Another group of rats were diet restricted between PND $17 \pm 1$ and PND 31 to match the body weights of BDL group rats, a method slightly modified from our previous study (29), and was designed as dietcontrol group (DC) $(n=12)$. This group rats were used to eliminate the nutritional effects on oxidative stress parameters and spatial performance. A fourth group rats with BDL for 2 wks and concomitant melatonin $500 \mu \mathrm{g} / \mathrm{kg} / \mathrm{d}$ (Sigma Chemical, St. Louis, MO; in ethanol and then in saline solution) intraperitoneal injection was designed as melatonin $(500 \mu \mathrm{g} / \mathrm{kg} / \mathrm{d})(\mathrm{M} 500)$ group $(n=12)$. A fifth group rats with BDL for 2 wks and concomitant melatonin $1000 \mu \mathrm{g} / \mathrm{kg} / \mathrm{d}$ intraperitoneal injection was designed as melatonin $(1000 \mu \mathrm{g} / \mathrm{kg} / \mathrm{d})(\mathrm{M} 1000)$ group $(n=10)$. Melatonin was administered at the dose of $500 \mu \mathrm{g} / \mathrm{kg} / \mathrm{d}$ according to previous studies $(23,27)$. However, higher dose of melatonin had been used in treating rat brain disorders; hence, melatonin $1000 \mu \mathrm{g} / \mathrm{kg} / \mathrm{d}$ was also tested in this study $(20,22)$. Considering the circadian pattern of pineal melatonin secretion, melatonin was administered intraperitoneally in the light phase.

Morris water maze. Fifty-four rats (SC, $n=11$; DC, $n=12$; BDL, $n=$ 10; M500, $n=11$, M1000, $n=10$ ) were evaluated for spatial learning and memory capabilities using a Morris water navigation protocol similar to our previously published methods (13). The Morris water maze is a test of spatial learning and memory in rodents and is considered to be dependent on both cortex and hippocampus $(30,31)$. The protocol included two phases of trials: spatial (submerged and not visible platform; 120 s duration; PND 27-30) and probe (platform removed; $60 \mathrm{~s}$ duration; PND 31). The submerged platform can assess the rat's acquisition memory of a spatial task. The probe trial assesses spatial bias in the rat's search pattern during retrieval (32). A circular steel tank $(180 \mathrm{~cm}$ diameter $\times 50 \mathrm{~cm}$ high $)$ was filled with water $\left(24 \pm 1^{\circ} \mathrm{C}\right)$ to a depth of $25 \mathrm{~cm}$. Room lights illuminated the pool and visual clues around the room were kept in constant location from day to day. Four points on the perimeter of the pool were designed north $(\mathrm{N})$, east $(\mathrm{E})$, south $(\mathrm{S})$, and west (W). A $10 \times 10 \mathrm{~cm}$ Plexiglas platform, onto which the rat could escape was positioned in the center of one of the quadrants, $1 \mathrm{~cm}$ below the water surface. A video camera was set above the center of the pool and connected to a videotraction system (Ethovision, Noldus, The Netherlands). Between PND 27 and 30, rats were trained for 16 trials (four trials a day) to locate and escape onto the submerged platform (acquisition trial). For each rat, the quadrant in which the platform was located remain constant, but the point of immersion into the pool varies between $\mathrm{N}, \mathrm{E}, \mathrm{S}$, and $\mathrm{W}$ in a quasi-random order for the 16 trials so that the rat was not able to predict the platform location from the point at which it was placed into the pool. On mounting the platform, the rats were given a $30 \mathrm{~s}$ rest period, after which the next trial was started. If the rat did not find the platform within $120 \mathrm{~s}$, it was guided to the platform for a $30 \mathrm{~s}$ rest. On day 5 (PND31), the platform was removed and the rats were released in the quadrant opposite the previous platform position. The rat was allowed $60 \mathrm{~s}$ of free swimming. The search pattern was tested to assess memory for platform location (probe trial) (32).

Measurement of plasma biochemistry parameters. After completion of the Morris water maze (PND 31), blood samples were collected by cardiocentesis. This was performed in the light phase of rats. Because of the limitation of blood samples, only half of rats ( $n=5-6 /$ group) were analyzed for aspartate aminotransferase (AST), alanine aminotransferase (ALT), creatinine, alkaline phosphatase, and ammonia according to the methods we have published previously (13). The remaining blood samples from six rats from each group and their corresponding tissue levels (liver, brain cortex, and hippocampus) were analyzed for malondialdehyde (MDA) and glutathione (GSH)/oxidized glutathione (GSSG) values for group comparisons.
Measurement of plasma and tissue MDA levels. MDA is the end product of peroxidation of polyunsaturated fatty acids. Therefore, MDA is widely used as an index of lipid peroxidation. Total MDA levels in the plasma and tissues were measured using the BIOXYTECHR MDA-586 kit from OXYRerearch (Portland, OR), according to the manufacturer's protocol. In short, the assay was based on the reaction of a chromogenic reagent, $N$-methyl-2phenylindole with MDA to form a colored carbocyanine dye with a maximum absorption at $586 \mathrm{~nm}$. Tissue samples were homogenized and protein concentration was determined by Bradford assay (Bio-Rad). Plasma and tissue homogenate samples were hydrolyzed by butylated hydroxytoluene at $\mathrm{pH}=$ $1-2$ at $60^{\circ} \mathrm{C}$ for $80 \mathrm{~min}$. Duplicate determinations in $100 \mu \mathrm{L}$ of plasma or tissue homogenate samples collected from all animals were made and the average of two measurements was used in subsequent statistical analysis of the data. The total MDA level was quantified spectrophotometrically at 586 $\mathrm{nm}$. The results were expressed as $\mu \mathrm{M}$ MDA per gram of protein.

Measurement of plasma total GSH and tissue reduced GSH to GSSG ratio. Plasma total GSH and tissue GSH/GSSG ratios, the main intracellular detoxifying molecules, were measured using a commercial kit (Glutathione Assay Kit II Cat No. 354103, Calbiochem), according to the manufacturer's protocol. In short, the assay uses a carefully optimized enzymatic recycling method, using GSH reductase, for the quantification of GSH. The GSH is quantified spectrophotometrically at $405 \mathrm{~nm}$. Quantification of GSSG, exclusive of GSH, is accomplished by first derivatizing GSH with 2-vinylpyridine. Tissue samples (liver and cortex) were homogenized on ice using a polytron homogenizer. The solution used for homogenization consisted of $50 \mathrm{mM}$ MES, which contains $0.4 \mathrm{M}$ 2-( $N$-morpholino) ethanesulphonic acid, $0.1 \mathrm{M}$ phosphate, and $2 \mathrm{mM}$ EDTA, pH 6.0 ( $1 \mathrm{~g}$ tissue per $5 \mathrm{~mL}$ MES buffer). The total homogenate was centrifuged at $4^{\circ} \mathrm{C}$ at $10,000 \times g$ for 15 min to obtain the supernatant for the assay of total GSH and GSSG. Duplicate determinations in $100 \mu \mathrm{L}$ of plasma or tissue homogenate samples collected from all animals were made and the average of two measurements was used in subsequent statistical analysis of the data. The GSH to GSSG ratios were calculated and expressed as GSH/GSSG ratio. Plasma GSSG levels were not detected because of the limitations of kit. We did not measure hippocampus GSH/GSSG ratios because of limited samples.

Statistical analysis. Morphologic parameters and biochemical parameters were analyzed by one-way ANOVA with post hoc Bonferroni test. Comparisons of the escape length during the 16 trials of place learning and probe trial of the Morris water maze were analyzed by one-way ANOVA with Bonferroni post hoc test and repeated measures. The swimming speed was analyzed with the nonparametric Kruskal-Wallis $H$ test. All analyses were performed using the Statistical Package for the Social Sciences (SPSS) software (VERSION 15) in a PC-compatible computer. Values were expressed as mean \pm $\mathrm{SE}$, and significance were defined as $p<0.05$ for all tests.

\section{RESULTS}

General morphologic features and plasma biochemistry. The mortality rate for the SC group, DC group, BDL group, M500 group, and M1000 group were 15.4\% (2/13), $0 \%(0 / 12)$, $23 \%(3 / 13), 8.3 \%(1 / 12)$, and $0 \%(0 / 10)$, respectively.

As shown in Table 1, SC group displayed higher body weights than those in other four groups. BDL rats at $2 \mathrm{wk}$ after surgery showed progressive jaundice, ascites, and hepatomeg-

Table 1. Morphological and plasma biochemical values in different experimental groups

\begin{tabular}{|c|c|c|c|c|c|}
\hline & $\mathrm{SC}(n=5)$ & $\mathrm{DC}(n=5)$ & $\operatorname{BDL}(n=5)$ & $\operatorname{M500}(n=6)$ & $\operatorname{M1000}(n=6)$ \\
\hline Body weight $(\mathrm{g})$ & $123.72 \pm 23.50 \S$ & $73.86 \pm 7.19 *$ & $73.19 \pm 14.27 *$ & $86.49 \pm 15.01 *$ & $97.68 \pm 20.22 *$ \\
\hline Liver weight $(\mathrm{g}) /$ Body weight $(\mathrm{g})$ & $0.048 \pm 0.005 \S$ & $0.040 \pm 0.004 \S$ & $0.070 \pm 0.01 *$ & $0.072 \pm 0.005^{*}$ & $0.078 \pm 0.005 * \S$ \\
\hline Brain weight $(\mathrm{g}) /$ Body weight $(\mathrm{g})$ & $0.012 \pm 0.002$ & $0.017 \pm 0.001 *$ & $0.015 \pm 0.002$ & $0.013 \pm 0.002$ & $0.012 \pm 0.002$ \\
\hline Direct bilirubin $(\mathrm{mg} \%)$ & $0.22 \pm 0.09 \S$ & $0.31 \pm 0.19 \S$ & $3.55 \pm 1.75^{*}$ & $3.40 \pm 1.55^{*}$ & $3.75 \pm 0.97 *$ \\
\hline Ammonia (mg\%) & $634 \pm 158.27$ & $628.5 \pm 156.73$ & $777.0 \pm 165.54$ & $706.6 \pm 193.33$ & $724.7 \pm 205.56$ \\
\hline Albumin $(\mathrm{g} \%)$ & $1.5 \pm 0.18 \S$ & $1.4 \pm 0.10 \S$ & $1.06 \pm 0.23 *$ & $1.0 \pm 0.12^{*}$ & $1.0 \pm 0.08^{*}$ \\
\hline Alk-Ptase (U/L) & $607.9 \pm 152.60$ & $344.2 \pm 52.26$ & $620.6 \pm 244.32$ & $816.0 \pm 311.73$ & $851.4 \pm 156.83$ \\
\hline Creatinine $(\mathrm{mg} \%)$ & $0.2 \pm 0.04$ & $0.2 \pm 0.04$ & $0.14 \pm 0.05$ & $0.2 \pm 0.05$ & $0.1 \pm 0.05$ \\
\hline
\end{tabular}

AST, aspartate aminotransferase; ALT, alanine aminotransferase; SC, sham-control; DC, diet-control; BDL, bile duct ligation; M500, melatonin (500 $\mu \mathrm{g} / \mathrm{kg} / \mathrm{d})$; M1000, melatonin $(1000 \mu \mathrm{g} / \mathrm{kg} / \mathrm{d}) . * p<0.05$, as compared with the SC; $\S p<0.05$, as compared with the BDL. 
aly. Hepatomegaly (represented as liver weight/body weight) was not prevented by melatonin therapy. As to plasma ammonia, albumin, alkaline phosphates, and creatinine levels, there were no significant differences between the five groups. BDL group had higher plasma direct and total bilirubin, AST, and ALT levels than SC group; however, the elevated total bilirubin, AST, and ALT levels in BDL group were partially prevented by melatonin therapy.

Morris water maze. Figure 1 showed the escape latency in the water maze as a function of days, and all the five groups displayed improvements over the $4 \mathrm{~d}$ of training. However, the main effect of group was significant $\left(F_{4,49}=4.655, p<0.05\right)$, and the post hoc Bonferroni test showed BDL-induced cholestasis in developing rats resulted in worse spatial memory. The improvement of impaired performance in BDL group rats was only seen in the jaundiced rats receiving melatonin at a dosage of $1000 \mu \mathrm{g} / \mathrm{kg} / \mathrm{d}$, not at a dosage of $500 \mu \mathrm{g} / \mathrm{kg} / \mathrm{d}$ (Fig. 1). In the probe trial, there was no significant difference in the percentage of time in the target quadrant (data not shown).

Plasma and tissue MDA levels. As shown in Figure 2 Panel $A$ and $B$, plasma MDA levels were higher in BDL group versus $\mathrm{SC}$ group, which was prevented by melatonin therapy. Similarly, MDA levels in the liver were higher in the BDL group versus SC group, which was completely prevented by melatonin therapy. These findings suggested that melatonin has an effect in decreasing systemic and hepatic lipid peroxidation caused by BDL-induced cholestasis. However, there were no significant differences of brain cortex and hippocampus MDA levels among all 5 groups (Fig. 2 Panel $C$ and $D$ ).

Plasma total GSH levels and tissue GSH/GSSG ratios. Plasma total GSH levels were not significantly different among SC, DC, BDL, and M500 groups (SC: $19.7 \pm 3.1 \mu \mathrm{M}$;

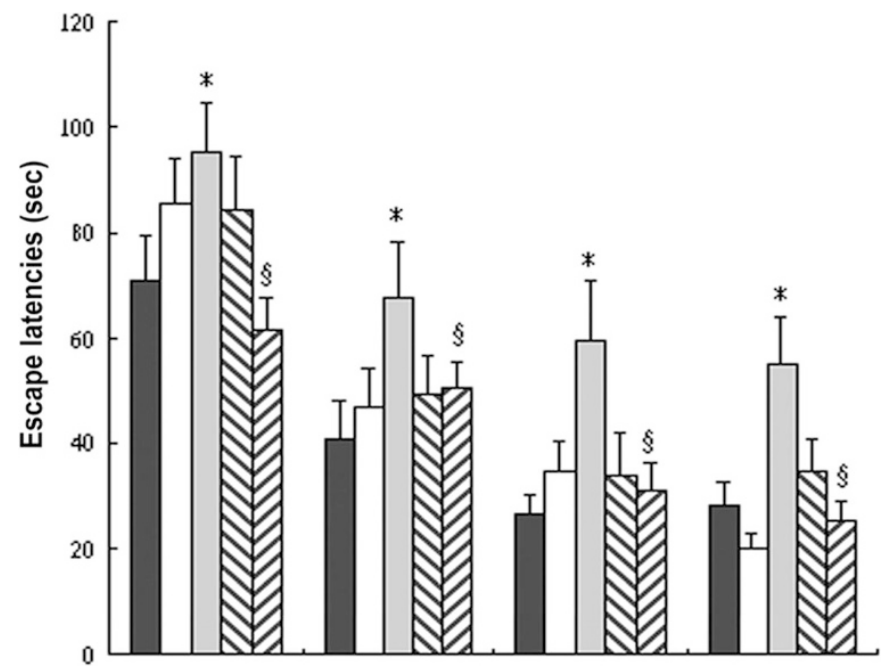

Figure 1. Latencies of escape to the platform in the Morris water maze (mean \pm SEM) for the sham-control (SC: $\square$ ), diet-control (DC: $\square$ ), jaundiced rats with bile duct ligation for $2 \mathrm{wk}$ (BDL: : : and jaundiced rats with therapy of melatonin at a dosage of $500 \mu \mathrm{g}$ (M500: $\mathbf{\nabla})$, or $1000 \mu \mathrm{g}$ (M1000: $\llbracket$ ). There was a significant main effect of the group $\left(F_{4,49}=4.655, p<0.05\right)$. The post hoc test revealed that BDL group rats had impaired spatial performance, when compared with either the SC or the DC group rats. And only the M1000 group rats could restore the spatial impairment and showed no difference with the SC group rats. $* p<0.05$, when compared with the $\mathrm{SC}$; $\S p<0.05$, when compared with the BDL.
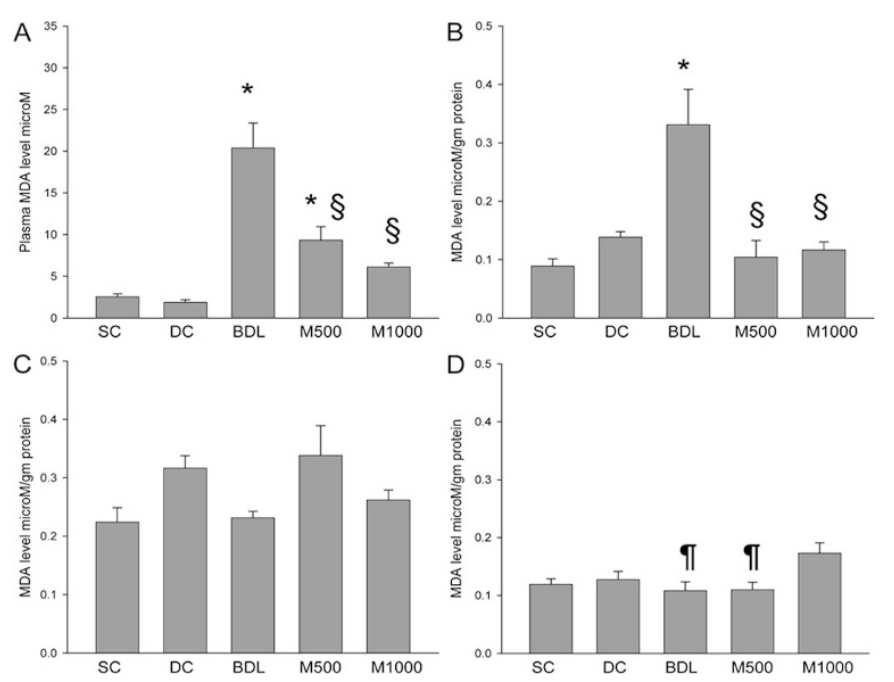

Figure 2. Plasma and tissue malondialdehyde (MDA) levels: $(A)$ plasma, $(B)$ liver, $(C)$ cortex, and $(D)$ hippocampus. $n=6$ /group. $\mathrm{DC}=$ diet-control; $\mathrm{BDL}=$ bile duct ligation; $\mathrm{M} 500=$ melatonin $500 \mu \mathrm{g} ; \mathrm{M} 1000=$ melatonin $1000 \mu \mathrm{g}$. ${ }^{*} p<0.05$, when compared with the SC; $\$ p<0.05$, when compared with the BDL. I $p<0.05$, when compared with M1000.

DC: $8.3 \pm 2.1 \mu \mathrm{M}$; BDL: $23.5 \pm 6.4 \mu \mathrm{M}$; M500:9 \pm 5.4 $\mu \mathrm{M})$. However, plasma total GSH levels were lower in M1000 versus $\mathrm{BDL}(5.2 \pm 2.1 \mu \mathrm{M}$ versus $23.5 \pm 6.4 \mu \mathrm{M}, p<0.05)$. Total GSH levels in the liver were higher in M1000 group than those in SC and M500 groups (Fig. 3 Panel A). The GSH/GSSG ratios in the liver were higher in M1000 group than those in the other four groups (Fig. 3 Panel C). There was no significant difference of cortex total GSH levels and GSH/ GSSG ratios among all five groups (Fig. 3 Panel $B$ and $D$ ).

\section{DISCUSSION}

The main findings of this study were 1) BDL-induced cholestasis in developing rat had worse spatial memory and
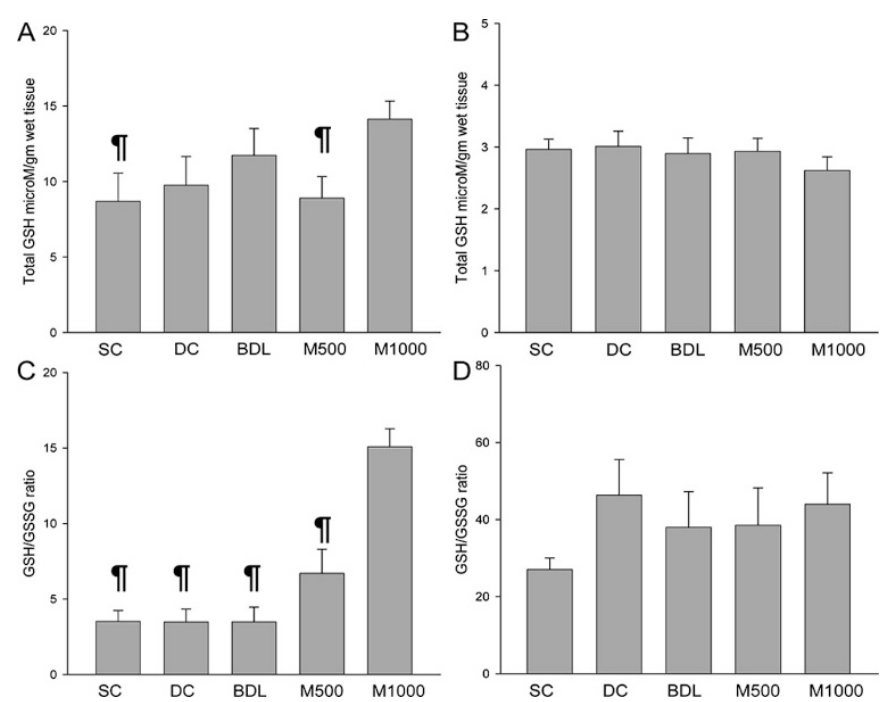

Figure 3. Total GSH level and GSH/GSSG ratio in the liver and cortex: $(A)$ liver total GSH, $(B)$ cortex total cortex, $(C)$ liver GSH/GSSG ratio, and $(D)$ cortex GSH/GSSG ratio. $n=6$ /group. $\mathrm{DC}=$ diet-control; $\mathrm{BDL}=$ bile duct ligation; $\mathrm{M} 500=$ melatonin $500 \mu \mathrm{g} ; \mathrm{M} 1000=$ melatonin $1000 \mu \mathrm{g} . \mathbb{I} p<$ 0.05 , when compared with M1000. 
increased liver and systemic oxidative stress as compared with jaundice-free rats; 2) melatonin treatment, in a dose-dependent manner, decreased liver and systemic oxidative stress, increased liver antioxidant activity, and improved spatial memory in developing rat with BDL-induced cholestasis.

The underlying mechanisms of increased systemic oxidative stress during cholestasis may be due to the retention of toxic bile acids, which stimulate the generation of reactive oxygen species (ROS) in hepatocytes and live mitochondria (3), and consequently hepatocellular necrosis and apoptosis (25). The increased ROS may cause target organs damage (e.g., brain, heart, and kidney) via systemic circulation $(4,6)$. GSH is a key intracellular antioxidant that is capable of interacting with free radicals to repair membrane lipid peroxides. Our data showed BDL-induced cholestasis in developing rat is characterized by accumulation of ROS in the liver is associated with reduced GSH/GSSG ratios, which is in line with adult BDL-induced cholestatic rat $(1,4,25,26,33)$. A relative decrease of GSH and an increase of GSSG in the liver, although both GSH and GSSG levels decrease in plasma, have been reported in adult BDL rats (33). Whereas GSH to GSSG ratio was decreased, suggesting the ratio is better than total GSH levels to represent cellular oxidative stress status. We found total GSH levels were not significantly decreased in the plasma, liver, and cortex of young BDL rats. M1000 group showed lowest plasma GSH levels whereas highest liver GSH levels and GSH/GSSG ratios suggesting melatonin may regulate the hepatic efflux of GSH to prevent liver damage. In contrast to adult BDL-induced cholestatic rat, we could not find a significant difference of MDA levels or GSH/GSSG ratios in either brain cortex or hippocampus $(4,6,27)$. Nevertheless, data from young BDL rats are scarce.

In the Morris water maze task, animals need to learn and use spatial strategies to locate a hidden platform that is dependent on both brain cortex and hippocampus $(30,31)$. In the present study, cholestatic rat had a poorer performance in acquisition memory when compared with jaundiced-free rat. Ammonia exerts a deleterious effect on cerebral function and is considered to play an important role in the pathogenesis of hepatic encephalopathy (34). In this regard, Jover et al.(35) added hyperammonia diet in BDL rat to simulate hepatic encephalopathy that occurs in humans. In this study, systemic oxidative stress, instead of ammonia, plays a role in the cognitive deficit in young rat with BDL-induced cholestasis.

ROS are involved in several diseases, including ischemic injury, Alzheimer's disease, Parkinson's disease, and Down's syndrome all of which affect cognitive processes (36-38). ROS can cause disruption of calcium homeostasis, membrane damage, and cell death (39), and has a detrimental effect on several key enzymes involved in glutamate and glucose transport $(40,41)$. All of the above-mentioned biologic effects can result in cognitive deficit. Although, we could not detect either MDA or GSH/GSSG differences in brain cortex or hippocampus, we postulate that other players in the oxidants/antioxidants system might play a role. Alternatively, other brain regions that are involved in spatial memory might account for the cholestasisinduced spatial dysfunction in our rats.
The present study clearly demonstrated that melatonin treatment, in a dose-dependent manner, abolished the increase in both plasma and liver MDA, probably in part by scavenging the very reactive hydroxyl, peroxyl and superoxide radicals. Furthermore, melatonin treatment restored liver GSH/GSSG levels significantly. There are some parallels between our study and those of adult rats (24-26). From the perspective of brain development, $17 \mathrm{~d}$ of age in rats is equal to young childhood and 27-31 d of age in rats is equal to school ages in human beings (42); hence, this model mimics some core features of biliary atresia patients who are complicated with cognitive problems despite Kasai operation (9). We extend these results to the developing rats and provide the first evidence that melatonin can improve cognition in young rats with cholestasis. Melatonin could protect DNA molecules, proteins, and membrane lipids from the harmful effects of free radicals, via the mechanism of electron donation with the generation of indolyl cation radical, a molecule also able to scavenge the toxic superoxide radical $(17,18)$. In the setting of experimental cholestasis, melatonin has been found to increase liver activity of catalase, superoxide dismutase, GSH peroxidase, GSH reductase and GSH transferase $(24,25)$, increase renal activity of reduced GSH (23), increase brain activity of superoxise dismutase, catalase, and GSH peroxidase (27). Although, we could not detect either MDA or GSH/GSSG ratio differences in brain cortex or hippocampus, these results suggest melatonin's action in brain may be via other mechanisms.

Finally, the findings of our present study demonstrate for the first time that melatonin, a safe and widely used drug, with its potent free radical scavenging and antioxidant properties, can counteract the BDL-induced systemic oxidative damages and spatial deficits in the developing rats.

\section{REFERENCES}

1. Huang YT, Hsu YC, Chen CJ, Liu CT, Wei YH 2003 Oxidative-stress-related changes in the livers of bile-duct-ligated rats. J Biomed Sci 10:170-178

2. Portincasa P, Grattagliano I, Testini M, Caruso ML, Wang QH, Moschetta A, Calamita G, Vacca M, Valentini AM, Renna G, Lissidini G, Palasciano G 2007 Parallel intestinal and liver injury during early cholestasis in the rat: modulation by bile salts and antioxidants. Free Radic Biol Med 42:1381-1391

3. Sastre J, Serviddio G, Pereda J, Minana JB, Arduini A, Vendemiale G, Poli G, Pallardo FV, Vina J 2007 Mitochondrial function in liver disease. Front Biosci 12:1200-1209

4. Ljubuncic P, Tanne Z, Bomzon A 2000 Evidence of a systemic phenomenon for oxidative stress in cholestatic liver disease. Gut 47:710-716

5. Assimakopoulos SF, Thomopoulos KC, Patsoukis N, Georgiou CD, Scopa CD, Nikolopoulou VN, Vagianos CE 2006 Evidence for intestinal oxidative stress in patients with obstructive jaundice. Eur J Clin Invest 36:181-187

6. Chroni E, Patsoukis N, Karageorgos N, Konstantinou D, Georgiou C 2006 Brain oxidative stress induced by obstructive jaundice in rats. J Neuropathol Exp Neurol 65:193-198

7. Feher J, Lengyel G, Blazovics A 1998 Oxidative stress in the liver and biliary tract diseases. Scand J Gastroenterol Suppl 228:38-46

8. Gilberstadt SJ, Gilberstadt H, Zieve L, Buegel B, Collier RO Jr., McClain CJ 1980 Psychomotor performance defects in cirrhotic patients without overt encephalopathy. Arch Intern Med 140:519-521

9. Stewart SM, Campbell RA, McCallon D, Waller DA, Andrews WS 1992 Cognitive patterns in school-age children with end-stage liver disease. J Dev Behav Pediatr 13:331-338

10. Erceg S, Monfort P, Hernandez-Viadel ML, Rodrigo R, Montoliu C, Felipo V 2005 Oral administration of sildenafil restores learning ability in rats with hyperammonemia and with portacaval shunt. Hepatology 41:299-306

11. Monfort P, Corbalan R, Martinez L, Lopez-Talavera J, Cordoba J, Felipo V 2001 Altered content and modulation of soluble guanylate cyclase in the cerebellum of rats with portacaval anastomosis. Neuroscience 104:1119-1125 
12. Monfort P, Munoz MD, Felipo V 2004 Hyperammonemia impairs long-term potentiation in hippocampus by altering the modulation of cGMP-degrading phosphodiesterase by protein kinase G. Neurobiol Dis 15:1-10

13. Huang LT, Hsieh CS, Chou MH, Chuang JH, Liou CW, Tiao MM, Lai MC 2004 Obstructive jaundice in rats: cause of spatial memory deficits with recovery after biliary decompression. World J Surg 28:283-287

14. Monfort P, Erceg S, Piedrafita B, Llansola M, Felipo V 2007 Chronic liver failure in rats impairs glutamatergic synaptic transmission and long-term potentiation in hippocampus and learning ability. Eur J Neurosci 25:2103-2111

15. Mendez M, Mendez-Lopez M, Lopez L, Aller MA, Arias J, Cimadevilla JM, Arias JL 2008 Spatial memory alterations in three models of hepatic encephalopathy. Behav Brain Res 188:32-40

16. Dumas TC 2005 Developmental regulation of cognitive abilities: modified composition of a molecular switch turns on associative learning. Prog Neurobiol 76:189211

17. Reiter R, Tang L, Garcia JJ, Garcia JJ, Munoz-Hoyos A 1997 Pharmacological actions of melatonin in oxygen radical pathophysiology. Life Sci 60:2255-2271

18. Reiter RJ 1998 Oxidative damage in the central nervous system: protection by melatonin. Prog Neurobiol 56:359-384

19. Rodriguez C, Mayo JC, Sainz RM, Antolin I, Herrera F, Martin V, Reiter RJ 2004 Regulation of antioxidant enzymes: a significant role for melatonin. J Pineal Res $36: 1-9$

20. Kerman M, Cirak B, Ozguner MF, Dagtekin A, Sucu R, Altuntas I, Delibas N 2005 Does melatonin protect or treat brain damage from traumatic oxidative stress? Exp Brain Res 163:406-410

21. Tutunculer F, Eskiocak S, Basaran UN, Ekuklu G, Ayvaz S, Vatansever U 2005 The protective role of melatonin in experimental hypoxic brain damage. Pediatr Int 47:434-439

22. Mohanan PV, Yamamoto HA 2002 Preventive effect of melatonin against brain mitochondria DNA damage, lipid peroxidation and seizures induced by kainic acid. Toxicol Lett 129:99-105

23. Cruz A, Padillo FJ, Tunez I, Munoz C, Granados J, Pera-Madrazo C, Montilla P 2001 Melatonin protects against renal oxidative stress after obstructive jaundice in rats. Eur J Pharmacol 425:135-139

24. Monitlla P, Cruz A, Padillo FJ, Tunez I, Gascon F, Munoz MC, Gomez M, Pera C 2001 Melatonin versus vitamin $\mathrm{E}$ as protective treatment against oxidative stress after extra-hepatic bile duct ligation in rats. J Pineal Res 31:138-144

25. Padillo FJ, Cruz A, Navarrete C, Bualance I, Briceno J, Galladro JI, Marchal T, Caballero R, Tunez I, Muntane J, Montilla P, Pera-Madrazo C 2004 Melatonin prevents oxidative stress and hepatocyte cell death induced by experimental cholestasis. Free Radic Res 38:697-704

26. Esrefoglu M, Gul M, Emre MH, Polat A, Selimoglu MA 2005 Protective effect of low dose of melatonin against cholestatic oxidative stress after common bile duct ligation in rats. World J Gastroenterol 11:1951-1956

27. Cruz A, Tunez I, Martinez R, Munoz-Castaneda JR, Ramirez LM, Recio M, Ochoa L, Arjona A, Montilla P, Muntane J, Padillo FJ 2007 Melatonin prevents brain oxidative stress induced by obstructive jaundice in rats. J Neurosci Res 85:36523656
28. Weiss MD, Wasdell MB, Bomben MM, Rea KJ, Freeman RD 2006 Sleep hygiene and melatonin treatment for children and adolescents with ADHD and initial insomnia. J Am Acad Child Adolesc Psychiatry 45:512-519

29. Huang LT, Lai MC, Wang CL, Wang CA, Yang CH, Hsieh CS, Liou CW, Yang SN 2003 Long-term effects of early-life malnutrition and status epilepticus: assessment by spatial navigation and CREB (Serine-133) phosphorylation. Brain Res Dev Brain Res 145:213-218

30. Morris R 1984 Developments of a water-maze procedures for studying spatial learning in the rat. J Neurosci Methods 11:47-60

31. Sloan HL, Good M, Dunnett SB 2006 Double dissociation between hippocampal and prefrontal lesions on an operant delayed matching task and a water maze reference memory task. Behav Brain Res 171:116-126

32. Jeltsch H, Bertrand F, Lazarus C, Cassel JC 2001 Cognitive performances and locomotor activity following dentate granule cell damage in rats: role of lesion exten and type of memory tested. Neurobiol Learn Mem 76:81-105

33. Purucker E, Winograd R, Roeb E, Matern S 1998 Glutathione status in liver and plasma during development of biliary cirrhosis after bile duct ligation. Res Exp Med (Berl) 198:167-174

34. Lockwood AH 2004 Blood ammonia and hepatic encephalopathy. Metab Brain Dis 19:345-349

35. Jover R, Rodrigo R, Felipo V, Insausti R, Saez-Valero J, Garcia-Ayllon MS, Surarez I, Candela A, Compan A, Esteban A, Cauli O, Auso E, Rodriguez E, Gutierrez A, Girona E, Erceg S, Berbel P, Perez-Mateo M 2006 Brain edema and inflammatory activation in bile duct ligated rats with diet-induced hyperammonemia: A model of hepatic encephalopathy in cirrhosis. Hepatology 43:1257-1266

36. Sayre LM, Smith MA, Perry G 2001 Chemistry and biochemistry of oxidative stres in neurodegenerative disease. Curr Med Chem 8:721-738

37. Perry G, Nunomura A, Hirai K, Zhu X, Perez M, Avila J, Castellani RJ, Atwood CS, Aliev G, Sayre LM, Takeda A, Smith MA 2002 Is oxidative damage the fundamental pathogenic mechanism of Alzheimer's and other neurodegenerative diseases? Free Radic Biol Med 33:1475-1479

38. Butterfield DA, Reed T, Newman SF, Sultana R 2007 Roles of amyloid beta-peptideassociated oxidative stress and brain protein modifications in the pathogenesis of Alzheimer's disease and mild cognitive impairment. Free Radic Biol Med 43:658677

39. Keller JN, Guo Q, Holtsberg FW, Bruce-Keller AJ, Mattson MP 1998 Increased sensitivity to mitochondrial toxin-induced apoptosis in neural cells expressing mutant presenilin-1 is linked to perturbed calcium homeostasis and enhanced oxyradical production. J Neurosci 18:4439-4450

40. Keller JN, Mattson MP 1998 Roles of lipid peroxidation in modulation of cellular signaling pathways, cell dysfunction, and death in the nervous system. Rev Neurosci 9:105-116

41. Lauderback CM, Hackett JM, Huang FF, Keller JN, Szweda LI, Markesbery WR, Butterfield DA 2001 The glial glutamate transporter, GLT-1, is oxidatively modified by 4-hydroxy-2-nonenal in the Alzheimer's disease brain: the role of Abeta1-42. J Neurochem 78:413-416

42. Andersen SL 2003 Trajectories of brain development: point of vulnerability or window of opportunity? Neurosci Biobehav Rev 27:3-18 